\title{
High-Intensity Low-Frequency Ultrasonic Treatment of Sulfated Polysaccharide from Brown Algae
}

\author{
Viktoria E. Suprunchuk* \\ North-Caucasus Federal University \\ Stavropol, Russian Federation
}

Received 15.11.2021, received in revised form 20.11.2021, accepted 01.12.2021

\begin{abstract}
This paper reports on an effective method for depolymerization of fucoidan in an aqueous medium using ultrasonic treatment. To assess the effect of this treatment, high-molecular-weight fucoidan was dispersed at a concentration of $10 \mathrm{mg} / \mathrm{ml}$ in deionized water and subjected to ultrasound at a frequency of $20 \mathrm{kHz}$ with varying intensity. The effect of high-intensity low-frequency ultrasound on the particle size of sulfated polysaccharides isolated from brown algae, where the minimum value of the average hydrodynamic diameter was $85.92 \pm 32.9 \mathrm{~nm}$, was studied. The dependence of the particle size of the polysaccharide on the intensity of ultrasonic exposure was revealed. The influence of this effect on the degree of sulfation of fucoidan has been determined. Shown the cavitation activity in the processing environment and discussed the reasons for its change.
\end{abstract}

Keywords: fucoidan, desulfation, depolymerization, ultrasonic treatment, cavitation, polysaccharide nanoparticles.

Acknowledgements. The author is grateful to ITMO and personally to V.V. Vinogradov for the opportunity to carry out work on the basis of SCAMT laboratories. This work was supported by a scholarship of the President of the Russian Federation for young scientists and graduate students № . CP-1758.2021.4 «Development of a nanobiocomposite tPA carrier for targeted high-performance thrombolytic therapy».

Citation: Suprunchuk, V.E. High-intensity low-frequency ultrasonic treatment of sulfated polysaccharide from brown algae, J. Sib. Fed. Univ. Chem., 2021, 14(4), 582-592. DOI: 10.17516/1998-2836-0265

(C) Siberian Federal University. All rights reserved

This work is licensed under a Creative Commons Attribution-NonCommercial 4.0 International License (CC BY-NC 4.0).

* Corresponding author E-mail address: vsuprunchuk@ncfu.ru 


\title{
Низкочастотная высокоинтенсивная \\ ультразвуковая обработка \\ сульфатированного полисахарида бурых водорослей
}

\author{
В. Е. Супрунчук \\ Северо-Кавказский федеральный университет \\ Российская Федерачия, Ставрополь
}

\begin{abstract}
Аннотация. В данной работе сообщается об эффективном методе деполимеризации фукоидана в водной среде с помощью ультразвуковой обработки. Для оценки эффекта такой обработки высокомолекулярный фукоидан был диспергирован в концентрации 10 мг/мл в деионизированной воде и подвергнут ультразвуковому воздействию с частотой в 20 кГЦ при варьировании интенсивности. Изучено влияние высокоинтенсивного низкочастотного ультразвука на размерность частиц сульфатированного полисахарида, выделенного из бурых водорослей, где минимальное значение среднего гидродинамического диаметра составило $85,92 \pm 32,9$ нм. Выявлена зависимость размера частиц полисахарида от интенсивности ультразвукового воздействия. Определено влияние такого воздействия на степень сульфатирования фукоидана. Показана кавитационная активность в среде обработки и обсуждены причины ее изменения.
\end{abstract}

Ключевые слова: фукоидан, десульфатирование, деполимеризация, ультразвуковая обработка, кавитация, полисахаридные наночастицы.

Благодарности. Автор выражает благодарность ИТМО и лично В. В. Виноградову за возможность проведения работы на базе лабораторий SCAMT. Работа выполнена при поддержке стипендии Президента РФ молодым ученым и аспирантам № СП-1758.2021.4 «Разработка нанобиокомпозитного носителя ТАП для таргетной высокоэффективной тромболитической терапии».

Цитирование: Супрунчук В.Е. Низкочастотная высокоинтенсивная ультразвуковая обработка сульфатированного полисахарида бурых водорослей / В. Е. Супрунчук // Журн. Сиб. федер. ун-та. Химия, 2021, 14(4). С. 582-592. DOI: $10.17516 / 1998-2836-0265$

\section{Введение}

Фукоидан - разветвленный сульфатированный геторополисахарид, выделяемый из клеточных стенок бурых морских водорослей и некоторых тканей морских беспозвоночных. Основным мономерным звеном фукоидана выступает L-фукоза, однако установлено присутствие в структуре также некоторых количеств остатков глюкозы, маннозы, ксилозы [1], галактозы $[2,3]$ глюкуроновой кислоты [4] и др. Сама структура фукоиданов не однородна, и можно выделить два наиболее часто встречаемых типа основной цепи. Первый тип представляет собой $1 \rightarrow 3$-связанные остатки $\alpha$ - L-фукопиранозы, второй тип - чередующиеся $1 \rightarrow 3$ - и $1 \rightarrow 4$-связанные остатки $\alpha$ - L-фукопиранозы [5]. Помимо сульфатных групп, которые, как правило, расположены

$$
-583-
$$


при С-2, С-3 и/или C-4 углеродном атоме фукозного кольца [6, 7], имеются ацетатные группы

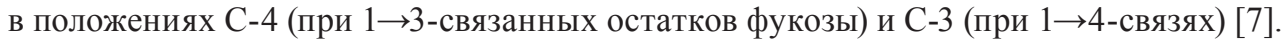

Интерес к проблеме исследования фукоидана растет, и в последнее десятилетие количество рецензируемых работ, посвященных изучению этого полисахарида, выросло в 3,6 раза. Фукоиданы обладают широким спектром биологической активности, в том числе проявляют противовирусное [8], противоопухолевое, противовоспалительное, антикоагулянтное [9] антитромбическое [10], антилипогенное и антиоксидантное [11, 12] действие, усиливают активность естественных киллеров, макрофагов, дендритных клеток и Т-клеток [13]; стимулируют гемопоэз [14] и т. д. Однако существуют ограничения его применения в связи с высокой молекулярной массой. Известно, что низкомолекулярные фукоиданы обладают улучшенными иммуномодулирующими, антиоксидантными свойствами [15-17], выраженным отбеливающим эффектом $[16,18]$. Снижение молекулярной массы необходимо в том числе и для увеличения реакционной способности полисахарида.

Сегодня существует множество подходов к деполимеризации полимеров, в том числе природного происхождения, и включают химические, ферментативные, физические методы. Для химического гидролиза наиболее часто используют кислоты и перекись водорода [19]. Ферментативные методы деполимеризации применяют, в частности, для деградации фукоиданов с образованием олигомеров $[20,21]$. Но в таких методах стоит вопрос о способах очистки конечного продукта, в особенности при использовании последнего для медицинских и фармацевтических целей. Кроме того, к недостаткам этих подходов можно отнести экологические проблемы, а также нежелательное разложение сопутствующих соединений, если речь идет о многокомпонентной системе [22]. Некоторые из способов, включающие применение концентрированных кислот (например, $\mathrm{HCl}$ ), имеют низкий выход и плохую воспроизводимость [22], а также приводят к образованию нежелательных мономеров и олигомеров [23] или разрушению химической структуры.

К физическим методам деградации относят ультразвуковое воздействие. Применение ультразвука широко изучено для деградации синтетических полимеров [24] (например, полиэтилена [25], полистирола [26], полиакрилметакрилатов) [27]. Для деградации природных полимеров ультразвуковое воздействие было использовано еще в 1933 г. [28] и в дальнейшем применялось для обработки декстрана [29], пектина [30], карбоксиметилцеллюлозы [31]. Ультразвуковое воздействие связано с колебаниями давления вследствие формирования кавитационных пузырьков. Кавитация является источником запуска многих химических процессов в системе, в том числе инициирует радикальные реакции. Кроме того, коллапс кавитационных пузырьков сопровождается физическими эффектами в виде ударных волн и горячих точек, которые вносят значительный вклад в деградацию полимера [32].

Ультразвуковая обработка приводит к снижению молекулярной массы и вязкости раствора полимера, но не сопровождается значительным изменениям химической структуры [32, 33]. При этом разрыв полимерной цепи осуществляется, как правило, в центральной точке или в самых слабых местах структуры, и скорость разложения увеличивается с ростом молекулярной массы полимера [33]. В целом, преимущество метода ультразвукового обработки заключается в его быстроте, мягкости и экологичности [34]. Тем не менее ультразвуковая деполимеризация фукоидана рассмотрена только для одного представителя, выделенного из морского огурца [35], причем 
физико-химические свойства получаемых частиц, в том числе влияние такой обработки на степень сульфатирования полисахарида, изучены не были. Поэтому целью работы явилось получение путем ультразвуковой обработки фукоидана его наноразмерной фракции, применимой для решения фармацевтических задач с изучением кавитационной активности в среде обработки.

\section{Материалы и методы}

Для ультразвуковой обработки использовали полисахарид, полученный из бурой водоросли Fucus vesiculosus по методике, представленной в работе [36], с некоторыми изменениями. Последующую очистку производили в соответствии с работой А. М. Урванцева, И.Ю. Бакунина, Н.Ю. Ким и др. [37]. Полученный полисахарид представлял собой светло-коричневый порошок, со степенью сульфатирования $22,79 \%$, с гидродинамическим размером частиц 10,4 $\pm 2,7 \mu$ м, ИК-спектр: 3326 см$^{-1}(-\mathrm{OH}), 1645$ см$^{-1}(\mathrm{C}-\mathrm{O}), 1217,1150$ и $1085 \mathrm{~cm}^{-1}(\mathrm{~S}=\mathrm{O}), 673 \mathrm{~cm}^{-1}$ $(-\mathrm{COOH}), 608 \mathrm{~cm}^{-1}(\mathrm{O}=\mathrm{S}=\mathrm{O})$.

В процессе ультразвуковой обработки был использован ультразвуковой процессор UIP1000hd мощностью 1000 Вт (Hielscher Ultrasonics GmbH, Германия). Все образцы были обработаны ультразвуковым воздействием в течение 40 мин с постоянной частотой 20 кГц и рабочей амплитудой 20, 40 и $80 \%$. Образцы объемом 100 мл и концентрацией фукоидана 10 мг/мл выдерживали при температуре $25 \pm 1,0{ }^{\circ} \mathrm{C}$. Во время обработки постоянство температурного режима поддерживалось с помощью ледяной бани с контролем температуры раствора, не превышающей 313 К, термодатчиком. Для работы подобрано невысокое значение концентрации полисахарида, так как известно, что степень деполимеризации полимеров уменьшается с увеличением концентрации раствора при сохранении подаваемой ультразвуковой мощности [25]. Измерение кавитации осуществлялось с помощью кавитометра.

После обработки для полученной фракции фукоидана была осуществлена оценка основных физико-химических показателей - гидродинамического диаметра, дзета-потенциала и степени сульфатирования. Наноразмерную фракцию отделяли путем фильтрования. Гидродинамический диаметр и электрохимический потенциал определяли с помощью Photocor Compact Z (ООО «Фотокор», Россия).

Определение степени сульфатирования осуществляли с помощью хлоридно-желатинового метода [38]. $\mathrm{BaCl}_{2}$-желатиновый раствор готовили следующим образом: 0,3 г желатина растворяли в 100 мл горячей воды $\left(60-70{ }^{\circ} \mathrm{C}\right)$, затем выдерживали в холодильнике при $4{ }^{\circ} \mathrm{C}$ в течение ночи. В полученный раствор желатина вводили 2 г $\mathrm{BaCl}_{2}$ и оставляли на 2-3 ч при комнатной температуре $[39,40]$. К 0,2 мл образца полисахарида вносили 3,8 мл 3\%-й трихлоруксусной кислоты и 1 мл $\mathrm{BaCl}_{2}$-желатиновый раствор инкубировали, перемешивая в течение 15 мин, с последующим измерением абсорбции при 360 нм [41] с помощью спектрофотометра Cary 8454 UV-Vis Diode Array System (Agilent Technologies Bayan Lepas Free, Малайзия). Бланк содержал 0,2 мл деионизированной воды. Количественное содержание сульфогрупп определяли по калибровочному графику, где в качестве стандарта был использован $\mathrm{Na}_{2} \mathrm{SO}_{4}$. Уравнение регрессии было следующим: $\mathrm{y}=0,029 \mathrm{x}+0,0692, \mathrm{R}^{2}=0,96, \mathrm{n}=6$. Степень сульфатирования полисахарида определялось как процентное содержание через соотношение массы сульфогрупп (m2) к массе полисахарида (m1).

$$
\% \text { сульфата }=(\mathrm{m} 2 / \mathrm{m} 1) \times 100 .
$$




\section{Результаты и обсуждение}

При ультразвуковой обработке водного раствора фукоидана ультразвуком с частотой 20 кГц полисахарид подвергается деградации, что приводит к достоверному снижению размера частиц биополимера. Отслеживание процессов деградации было осуществлено путем определения изменения размера частиц фукоидана с помощью метода динамического рассеяния света.

При определении зависимости гидродинамического диаметра частиц наноразмерной фракции фукоидана от интенсивности ультразвукового воздействия была получена серия образцов. Для этого водные растворы фукоидана подвергали ультразвуковому воздействию при варьировании интенсивности ультразвуковой волны от 100 до 133 и $200 \mathrm{BT} / \mathrm{cm}^{2}$, при этом обработку осуществляли в течение 40 мин при наличии охлаждающей рубашки и контроле температурного режима, не превышающего 313 К. Важно, что данный температурный режим обусловлен тем, что коллапсирующие пузырьки могут значительно увеличить температуру (до 5000 К) как в самом схлопывающемся пузыре, так и в области в непосредственной близости от пузыря [42]. Повышение температуры при ультразвуковом воздействии оказывает негативное влияние на скорость химических реакций, в том числе на скорость деполимеризации [25]. При повышении температуры раствора в кавитационные пузырьки попадают пары растворителя, что оказывает амортизирующий эффект при их схлопывании, что в свою очередь ведет к снижению силы ударной волны $[25,26]$. В целом, сонохимические реакции имеют более высокие скорости при низких температурах [26].

В соответствии с рис. 1 наблюдается возрастание доли наноразмерной фракции фукоидана с ростом интенсивности ультразвукового воздействия. Средний гидродинамический диаметр полученных частиц, как и в случае молекулярной массы, имеет определенное значение для заданной интенсивности. Однако невысокий выход наноразмерной фракции фукоидана может быть связан с конформацией макромолекул полисахарида. Так, известно, что у разветвленных полисахаридов формы «клубка» разрыв цепи более затруднен, чем у линейных «палочковид-

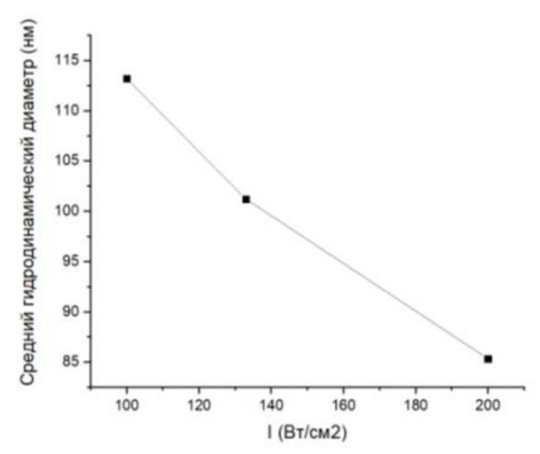

A

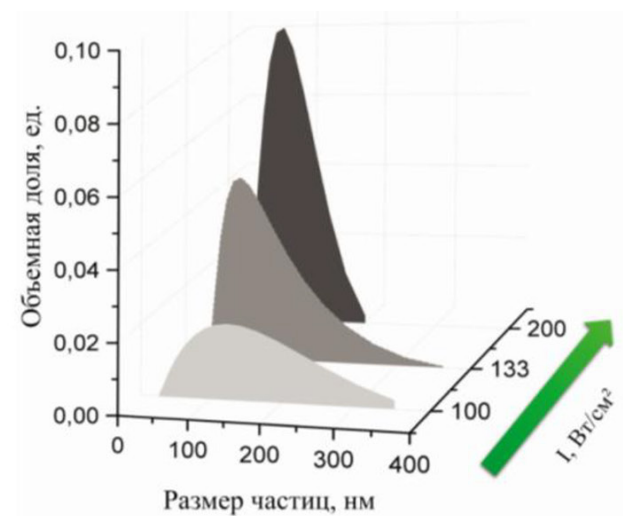

Б

Рис. 1 График зависимости размера (А) и объемной доли (Б) частиц фукоидана от интенсивности ультразвукового воздействия

Fig. 1. Dependence of the size (A) and volume fraction (B) of fucoidan particles on ultrasonic exposure 
ных» макромолекул (например, хитозана), вследствие того, что линейная конформация приводит к накоплению «тянущих сил» вдоль всей цепи [43]. Возможно, что данное затруднение преодолевается при повышении интенсивности ультразвуковой волны.

Дальнейшая обработка раствора полисахарида во всех случаях (более 80 мин) не привела к заметному уменьшению размеров частиц и доли наноразмерной фракции, поэтому является нецелесообразной. При увеличении рабочей амплитуды с 20 до 80 \% размер получаемых частиц фукоидана сократился в 1,3 раза (рис. 1), что может быть связано с достаточным временем для образования кавитаций. Минимальный средний гидродинамический диаметр частиц наблюдается в результате ультразвукового воздействия с интенсивностью $200 \mathrm{Bт} / \mathrm{cm}^{2}$ и составил $85,92 \pm 32,9$ нм.

Таким образом, подтверждается тезис о том, что предел размера частиц, как и молекулярной массы, находится в прямой зависимости от интенсивности ультразвуковой волны.

Значительной характеристикой при определении влияния ультразвукового воздействия на фукоидан является определение степени сульфатирования обработанного полисахарида. Степень сульфатирования - важная характеристика при проявлении фукоиданом антикоагулянтного или антипролиферативного действия [44].

В работе был использован хлоридно-желатиновый метод определения содержания этерифицированной серы, который осуществлен с использованием негидролизованного фукоидана, что позволило провести оценку свободной («доступной») серы, способной вступать в химические взаимодействия, что важно при проявлении биологических свойств.

Как показано на рис. 2, в результате сонохимической обработки степень сульфатирования снижается на 3,63\%. Снижение степени сульфатирования сопровождается изменением электрохимического потенциала с $-10,54$ до $-7,5$ мВ. В результате можно сделать вывод о чувствительности сульфогрупп к ультразвуковому воздействию.

Важным параметром, характеризующим ультразвуковой процесс, выступает такое явление, как кавитация. Кавитация служит источником запуска многих химических процессов

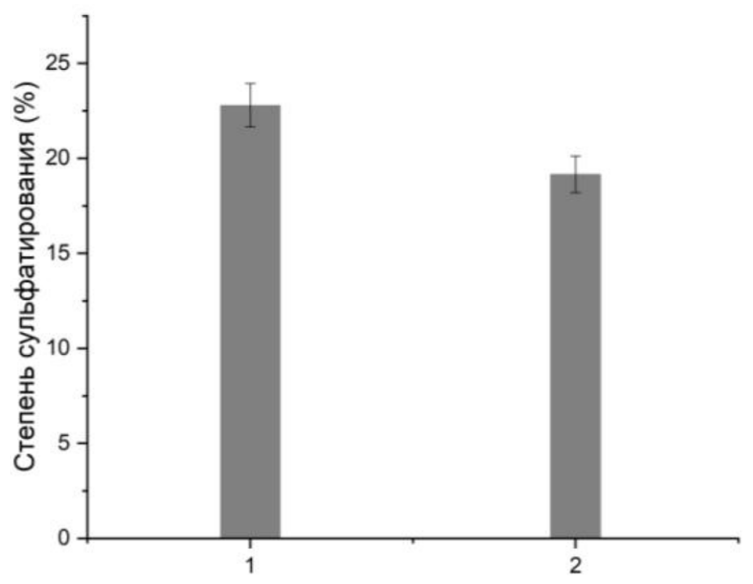

Рис. 2. Степень сульфатирования исходного фукоидана (1) и обработанного ультразвуком с частотой 20 кГц в течение 40 мин (2)

Fig. 2. Degree of sulfation of native fucoidan (1), sonicated at a frequency of $20 \mathrm{kHz}$ for $40 \mathrm{~min}$ (2)

$$
-587-
$$


в системе, в частности инициирует радикальные реакции. В результате схлопывания кавитационного пузырька создается ударная волна с образованием акустического потока, приводящего к формированию турбулентности из-за непрерывного формирования и схлопывания полостей в системе, при этом создаются ударные волны и горячие точки, которые вносят значительный вклад в деградацию полимера [32]. Поэтому помимо входных параметров ультразвука, используемого для генерации кавитации, необходимо осуществление измерения выходных сигналов, создаваемых кавитацией. В работе измерение кавитации осуществлялось с помощью кавитометра, действие которого основано на обработке спектра кавитационного шума, так называемого белого шума, принимаемого широкополосным гидрофоном, с последующим преобразованием акустического сигнала в электрический. Чем интенсивнее ударная волна, тем более широк спектр кавитационного шума и больше электрический сигнал. Измерение кавитационной активности в виде электрического сигнала осуществлялось во время ультразвукового воздействия на раствор полисахарида.

Исследование показало, что в ходе ультразвукового воздействия наблюдается снижение кавитации в течение первых 500 мс (рис. $3 A$ ). Однако затем на 6-й минуте воздействия наблюдается усиление электрического сигнала, то есть рост кавитации до максимального значения $14,1 \pm 0,37$ мВ, и затем этот сигнал снижается до невысоких значений от $5,2 \pm 0,47$ до 4,4 40,28 мВ в пределах 40-й минуты (рис. 3Б).

Сильный электрический сигнал в течение первых миллисекунд, возможно, обусловлен высокой концентрацией газовых пузырьков в растворе. Так, одновременно осуществляется схлопывание изначально существующих газовых пузырьков в системе, а также вновь генерируемых [45]. Однако впоследствии высокая концентрация кавитационных пузырьков приводит к их кластеризации. В результате кластеризации пузырьки мешают друг другу схлопываться из-за экранирования, что вызывает изменение акустической эмиссии, а сохранение пузырьков, в свою очередь, может блокировать передачу звука через жидкость к гидрофону [46], в результате чего электрический сигнал снижается.

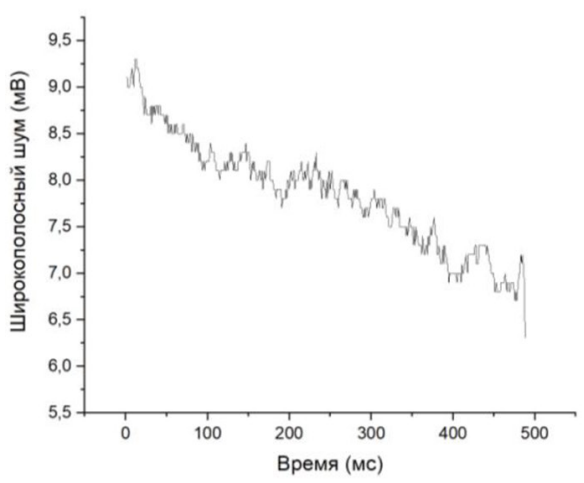

A

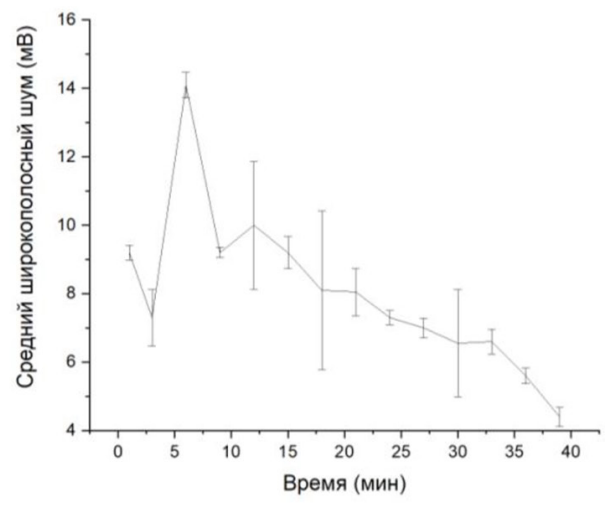

Б

Рис. 3. График изменения кавитационной активности при ультразвуковом воздействии в течение первых 500 мс (А) и 40 мин (Б)

Fig. 3. Changes in the activity of cavitation under ultrasound exposure during the first $500 \mathrm{~ms}(\mathrm{~A})$ and $40 \mathrm{~min}$ (B) 
Таким образом, наблюдается первоначальное повышение с последующим снижением кавитационной активности.

\section{Заключение}

В работе выявлено действие высокоинтенсивной низкочастотной ультразвуковой обработки на физическо-химические характеристики фукоидана. Исследована квитационная активность в среде обработки, а также зависимость размера частиц полисахарида от интенсивности ультразвукового воздействия. Данный способ деструкции полисахарида позволил получить наноразмерную фракцию фукоидана со средним гидродинамическим диаметром частиц 85,92 32,9 нм. Преимуществом ультразвукового способа получения наноразмерной фракции фукоидана является возможность прямого использования полученного раствора, исключая стадию очистки, в создании лекарственных форм. Таким образом, ультразвуковая обработка фукоидана представляет собой экологически чистый, дешевый и эффективный метод модификации этого биополимера; его можно использовать для получения низкомолекулярных фракций фукоидана с предсказуемыми характеристиками.

Дальнейшая работа будет направлена на подбор способа создания носителя с последующей иммобилизацией тромболитического агента с использованием полученной наноразмерной фракции фукоидана.

\section{Список литературы / References}

1. Cong, Q., Chen, H., Liao, W., Xiao, F., Wang, P., Qin, Y., Dong, Q., Ding, K. Structural characterization and effect on anti-angiogenic activity of a fucoidan from Sargassum fusiforme. Carbohydrate Polymers. 2016. Vol. 136. P. 899-907.

2. Cuong, H. D., Thuy, T. T. T., Huong, T. T., Ly, B. M., Van, T. T. T. Structure and hypolipidaemic activity of fucoidan extracted from brown seaweed Sargassum henslowianum. Natural Product Research. 2015. Vol. 29 (5). P. 411-415.

3. Bilan, M. I., Grachev, A. A., Shashkov, A. S., Nifantiev, N. E., Usov, A. I. Structure of a fucoidan from the brown seaweed Fucus serratus L. Carbohydrate Research. 2006. Vol. 341 (2). P. 238-245.

4. Ale, M. T., Maruyama, H., Tamauchi, H., Mikkelsen, J. D., Meyer, A. S. Fucoidan from Sargassum sp. and Fucus vesiculosus reduces cell viability of lung carcinoma and melanoma cells in vitro and activates natural killer cells in mice in vivo. International Journal of Biological Macromolecules. 2011. Vol. 49 (3). P. 331-336.

5. Khatuntseva, E. A., Ustuzhanina, N. E., Zatonskii, G. V., Shashkov, A. S., Usov, A. I., Nifant'ev, N.E. Synthesis, NMR and conformational studies of fucoidan fragments 1: 1 desulfated 2,3and 3,4-branched trisaccharide fragments and constituting disaccharides. Journal of Carbohydrate Chemistry. 2000. Vol. 19 (9). P. 1151-1173.

6. Yao, Y., Yim, E. K. F. Fucoidan for cardiovascular application and the factors mediating its activities. Carbohydrate Polymers. 2021. Vol. 270. P. 118347.

7. Li, B., Lu, F., Wei, X., Zhao, R. Fucoidan: structure and bioactivity. Molecules. 2008, Vol. 13 (8), P. 1671-1695. 
8. Wang, W., Wu, J., Zhang, X., Hao, C., Zhao, X., Jiao, G., Shan, X., Tai, W., Yu, G. Inhibition of influenza A virus infection by fucoidan targeting viral neuraminidase and cellular EGFR pathway. Scientific Reports. 2017. Vol. 7. P. 1-14.

9. Colliec, S., Fischer, A. M., Tapon-Bretaudiere, J., Boisson, C., Durand, P., Jozefonvicz, J. Anticoagulant properties of a fucoidan fraction. International Journal of Biological Macromolecules. 2018. Vol. 64 (2). P. 143-154.

10. Cui, K.; Tai, W.; Shan, X.; Hao, J.; Li, G.; Yu, G. Structural Characterization and AntiThrombotic Properties of Fucoidan from Nemacystus Decipiens. International Journal of Biological Macromolecules. 2018, Vol. 120, P. 1817-1822.

11. Wang, S., Huang, C., Chen, C., Chang, C. Isolation and purification of brown algae fucoidan from Sargassum siliquosum and the analysis of anti-lipogenesis activity. Biochemical Engineering Journal. 2021. Vol. 165. P. 107798.

12. Wang, S., Huang, C., Chen, C., Chang, C., Huang, C., Dong, C., Chang, J. Structure and biological activity analysis of fucoidan isolated from Sargassum siliquosum. ACS omega. 2020. Vol. 5 (50). P. 32447-32455.

13. Etman S.M., Elnaggar Y.S.R., Abdallah O.Y. Fucoidan, a natural biopolymer in cancer combating: From edible algae to nanocarrier tailoring. International Journal of Biological Macromolecules. 2020. Vol. 147. P. 799-808.

14. Li, C., Niu, Q., Li, S., Zhang, X., Liu, C., Cai, C., Li, G., Yu, G. Fucoidan from sea cucumber Holothuria polii: Structural elucidation and stimulation of hematopoietic activity. International Journal of Biological Macromolecules. 2020. Vol. 154. P. 1123-1131.

15. Borazjani, N. J., Tabarsa, M., You, S. G., Rezaei, M. Improved immunomodulatory and antioxidant properties of unrefined fucoidans from Sargassum angustifolium by hydrolysis Journal of Food Science and Technology. 2017. Vol. 54 (12). P. 4016-4025

16. Fernando, I. P. S., Kim, D., Nah, J. W., Jeon, Y. J. Advances in functionalizing fucoidans and alginates (bio)polymers by structural modifications: A review. Chemical Engineering Journal. 2019. Vol. 355. P. 33-48.

17. Yu, J., Li, Q., Wu, J., Yang, X., Yang, S., Zhu, W., Liu, Y. Fucoidan Extracted From Sporophyll of Undaria pinnatifida Grown in Weihai, China - Chemical Composition and Comparison of Antioxidant Activity of Different Molecular Weight Fractions. Frontiers in Nutrition. 2021. Vol. 8. P. 1-11.

18. Park E.J., Choi J. il. Melanogenesis inhibitory effect of low molecular weight fucoidan from Undaria pinnatifida. Journal of Applied Phycology. 2017. Vol. 29(5). P. 2213-2217.

19. Qin C.Q., Du Y.M., Xiao L. Effect of hydrogen peroxide treatment on the molecular weight and structure of chitosan. Polymer Degradation and Stability. 2002. Vol. 76 (2). P. 211-218.

20. Silchenko, A. S., Rasin, A. B., Kusaykin, M. I., Kalinovsky, A. I., Miansong, Z., Changheng, L., Malyarenko, O., Zueva, A. O., Zvyagintseva, T. N., Ermakova, S.P. Structure, enzymatic transformation, anticancer activity of fucoidan and sulphated fucooligosaccharides from Sargassum horneri. Carbohydrate Polymers. 2017. Vol. 175. P. 654-660.

21. Hwang, P., Yan, M., Kuo, K., Phan, N. N., Lin, Y. A mechanism of low molecular weight fucoidans degraded by enzymatic and acidic hydrolysis for the prevention of UVB damage // Journal of Applied Phycology. 2016. Vol. 29. P. 521-529. 
22. Cui R., Zhu F. Ultrasound modified polysaccharides: A review of structure, physicochemical properties, biological activities and food applications. Trends in Food Science and Technology. 2021. Vol. 107. P. 491-508.

23. Kulicke, W.-M., Clasen, C., Lohman, C. Characterization of water-soluble cellulose derivatives in terms of the molar mass and particle size as well as their distribution. Macromolecular Symposia. 2005. Vol. 223 (1). P. 151-174.

24. Basedow A.M., Ebert K.H. Ultrasonic degradation of polymers in solution. Advances in Polymer Science. 1970. P. 83-148.

25. Desai V., Shenoy M.A., Gogate P.R. Ultrasonic degradation of low-density polyethylene. Chemical Engineering and Processing: Process Intensification. 2008. Vol. 47 (9-10). P. 1451-1455

26. Price G.J., Paul F. S. Ultrasonic degradation of polymer solutions: 2. The effect of temperature, ultrasound intensity and dissolved gases on polystyrene in toluene. Polymer. 1993. Vol. 34 (19). P. 41114117.

27. Malhotra S.L. Ultrasonic solution degradations of poly (alkyl methacrylates). Journal of Macromolecular Science - Chemistry. 2006. Vol. 23 (6). P. 729-748.

28. Szent-Guorgyi A. Chemical and biological effects of ultra-sonic radiation. Nature. 1933. Vol. 131 (3304). P. 278-278.

29. Mason J., Cuthbert C., Brookfield A. Effect of ultrasound on the degradation of aqueous native dextran. Ultrasonics Sonochemistry. 1995. Vol. 2 (1). P. 1-3.

30. Tiwari, B. K., Muthukumarappan, K., Donnell, C. P. O., Cullen, P. J., Muthukumarappan, K., Donnell, C. P. O., Cullen, P. J., Tiwari, B. K., Muthukumarappan, K., Donnell, C. P. O. Rheological properties of sonicated guar, xanthan and pectin dispersions. International Journal of Food Properties. 2010. Vol. 13 (2). P. 223-233.

31. Pentti P. Ultrasonic degradation of aqueous carboxymethylcellulose: Effect of viscosity, molecular mass, and concentration. Ultrasonics Sonochemistry. 2008. Vol. 15. P. 644-648.

32. Gogate P.R., Prajapat A.L. Depolymerization using sonochemical reactors: A critical review Ultrasonics Sonochemistry. 2015. Vol. 27. P. 480-494.

33. Yin, J. Y., Ma, L. Y., Siu, K. C., Wu, J. Y. Effects of ultrasonication on the conformational, microstructural, and antioxidant properties of konjac glucomannan. Applied Sciences. 2019. Vol. 9. (3). P. 461

34. Yan, J.-K., Wang, Y.-Y., Ma, H.-L., Wang, Z.-B. Ultrasonic effects on the degradation kinetics, preliminary characterization and antioxidant activities of polysaccharides from Phellinus linteus mycelia. Ultrasonics Sonochemistry. 2016. Vol. 29. P. 251-257.

35. Guo, X., Ye, X., Sun, Y., Wu, D., Wu, N., Hu, Y., Chen, S. Ultrasound effects on the degradation kinetics, structure, and antioxidant activity of sea cucumber fucoidan. Journal of Agricultural and Food Chemistry. 2014. Vol. 62 (5). P. 1088-1095.

36. Zvyagintseva, T. N., Shevchenko, N. M., Popivnich, I. B., Isakov, V. V., Scobun, A. S., Sundukova, E. V., Elyakova, L. A. A new procedure for the separation of water-soluble polysaccharides from brown seaweeds. Carbohydrate Research. 1999. Vol. 322 (1-2). P. 32-39.

37. Урванцева, А. М., Бакунина, И. Ю., Ким, Н. Ю., Исаков, В. В., Глазунов, В. П., Звягинцева, Т.Н. Выделение очищенного фукоидана из природного комплекса с полифенолами и его характеристика. Химия растительного сырья. 2004. (3). P. 15-24. [Urvantseva, А. M., Bakunina, 
I. Yu., Kim, N. Yu., Isakov, V. V., Glazunov, V. P., Zvyagintseva, T.N. Isolation of purified fucoidan from a natural complex with polyphenols and its characteristic. Khimija Rastitel'nogo Syr'ja. 2004. (3). P. 15-24. (In Russ.)].

38. Dodgson K. S.; Price R. G. A note on the determination of the ester sulphate content of sulphated polysaccharides. Biochemical Journal. 1962. Vol. 84 (1). P. 106-110.

39. Sakthivel, R., Pandima Devi, K. Evaluation of physicochemical properties, proximate and nutritional composition of Gracilaria edulis collected from Palk Bay. Food Chemistry. 2015. Vol. 174. P. $68-74$.

40. Saito, H., Yamagata, T., Suzuki, S. Enzymatic methods for the determination of small quantities of isomeric chondroitin sulfates. Journal of Biological Chemistry. 1968. Vol. 243 (7). P. $1536-1542$.

41. Gracher, A. H. P., Santana, A. G,; Cipriani, T. R., Iacomini, M. A procoagulant chemically sulfated mannan. Carbohydrate Polymers. 2016. Vol. 136. P. 177-186.

42. Leonelli, C., Mason, T.J. Microwave and ultrasonic processing: Now a realistic option for industry. Chemical Engineering \& Processing: Process Intensification. 2010. Vol. 49 (9). P. 885-900.

43. Czechowska-Biskup, R., Rokita, B., Lotfy, S., Ulanski, P., Rosiak, J.M. Degradation of chitosan and starch by 360-kHz ultrasound. Carbohydrate Polymers. 2005. Vol. 60 (2). P. 175-184.

44. Flórez-fernández N., López-garcía M., González-muñoz M. J. Ultrasound-assisted extraction of fucoidan from Sargassum muticum. Journal of Applied Phycology, 2017. Vol. 29. P. 1553-1561.

45. Hallow, D. M., Mahajan, A. D., McCutchen, T. E., Prausnitz, M.R. Measurement and correlation of acoustic cavitation with cellular bioeffects. Ultrasound in Medicine and Biology. 2006. Vol. 32 (7.) P. 1111-1122.

46. Verhaagen B., Fernández Rivas D. Measuring cavitation and its cleaning effect. Ultrasonics Sonochemistry. 2016. Vol. 29. P. 619-628.

47. Wang, Y., Xing, M., Cao, Q., Ji, A., Liang, H., Song, S. Biological activities of fucoidan and the factors mediating its therapeutic effects: a review of recent studies. Marine Drugs. 2019. Vol. 17 (3). P. 183. 